\title{
Hausdorff Dimensions of Zero-Entropy Sets of Dynamical Systems with Positive Entropy
}

\author{
Xiongping Dai ${ }^{1}$ and Yunping Jiang ${ }^{2,3}$
}

Received March 15, 2005; accepted June 13, 2005

\begin{abstract}
Suppose that $(X, T)$ is a compact positive entropy dynamical system which we mean that $X$ is a compact metric space and $T: X \rightarrow X$ is a continuous transformation of $X$ and the topological entropy $h(T)>0$. A point $x \in X$ is called a zero-entropy point provided $h\left(T ; \overline{\operatorname{Orb}_{+}(x)}\right)=0$, where $\operatorname{Orb}_{+}(x)=\left\{T^{n}(x) \mid n \in\right.$ $\left.\mathbb{Z}_{+}\right\}$is the forward orbit of $x$ under $T$ and $\overline{\operatorname{Orb}_{+}(x)}$ is the closure. Let $\mathcal{E}^{0}(X, T)$ denote the set of all zero-entropy points. Naturally, one would like to ask the following important question:
\end{abstract}

$$
\text { How big is } \mathcal{E}^{0}(X, T) \text { for a dynamical system? }
$$

In this paper, we answer this question. More precisely, we prove that if, furthermore, $(X, T)$ is locally expanding, then the Hausdorff dimension of $\mathcal{E}^{0}(X, T)$ vanishes.

KEY WORDS: Zero-entropy point; zero-entropy set; entropy; Hausdorff dimension; locally expanding map; substitution sequence.

\section{INTRODUCTION}

In the study of dynamical systems, one always hopes to pay more attention to an "important part" of a dynamical system. What is an "important part"? This really depends upon his viewpoint. For a given dynamical system $(X, T)$, for example, from the viewpoint of ergodic theory, the generic

\footnotetext{
${ }^{1}$ Department of Mathematics, Nanjing University, Nanjing 210093, P. R. China; e-mail: xpdai@nju.edu.cn

${ }^{2}$ Department of Mathematics. Queens College of CUNY, Flushing, NY 11367, and Department of Mathematics, CUNY Graduate School, New York, NY 10016, USA.

${ }^{3}$ Academy of Mathematics and System Sciences. Chinese Academy of Sciences, Beijing 100080, P. R. China; e-mail: yunqc@forbin.qc.edu
} 
point set $G(T)$ consisting of all points $x \in X$ at which limits

$$
\lim _{n \rightarrow \infty} \frac{1}{n} \sum_{i=0}^{n-1} \varphi\left(T^{i} x\right)=\varphi^{*}(x) \quad\left(\forall \varphi \in C^{0}(X, \mathbb{R})\right)
$$

exist is important. On the other hand, from the viewpoint of topology, the nonwandering point set $\Omega(T)$ and the $\omega$-limit set $L(T)$ are both important. Each of the subsystems $\left(G(T), T_{\mid G(T)}\right),\left(\Omega(T), T_{\mid \Omega(T)}\right)$, and $\left(L(T), T_{\mid L(T)}\right)$ contains almost all important dynamical behaviors of the original dynamical system $(X, T)$. However, we still would like to ask the following question:

Are such subsystems "really" simpler or smaller than the original $(X, T)$ ?

Notice that it is well known that $G(T), \Omega(T)$, and $L(T)$ are all totally measure 1 from the viewpoint of ergodic theory! So they are not really smaller than $X$ from the measure-theoretical point of view. Our important goal in this paper is to find another small subsystem which remains the most important dynamical information of the original system entropy.

In the rest of the paper, we assume that $X$ is a compact metric space with the metric structure $d$. For any compact subset $Y \subseteq X$, let $h(T ; Y)$ denote the standard topological entropy of $T$ restricted on $Y$ (see, e.g., ref. 7 for the definition of the topological entropy of a dynamical system).

By a compact system $(X, T)$ defined on $X$, we mean $T: X \rightarrow X$ is a continuous transformation of $X$. A compact dynamical system $(X, T)$ is called positive entropy if the topological entropy $h(T)$ is positive. Let $\mathbb{Z}_{+}$ mean the set of all nonnegative integers and let

$$
\operatorname{Orb}_{+}(x)=\left\{T^{n}(x) \mid n \in \mathbb{Z}_{+}\right\}
$$

denote the forward orbit of $x$ under $T$ and $\overline{\operatorname{Orb}_{+}(x)}$ denote the closure. In general, a compact positive entropy dynamical system $(X, T)$ contains chaotic orbits. Therefore, we would like to make the following definition.

Definition 1. For any given compact system $(X, T)$, a point $x \in X$ is called a zero-entropy point if $h\left(T ; \overline{\operatorname{Orb}_{+}(x)}\right)=0$.

Furthermore, we define

$$
\begin{aligned}
& \widehat{O}(X, T)=\left\{x \in X \mid h\left(T ; \overline{\mathrm{Orb}_{+}(x)}\right)=0\right\}, \\
& \mathcal{E}^{0}(X, T)=\bigcup\left\{\overline{\operatorname{Orb}_{+}(x)} \mid x \in \widehat{O}(X, T)\right\}
\end{aligned}
$$


and call $\mathcal{E}^{0}(X, T)$ the zero-entropy set of $(X, T)$. Clearly, $\mathcal{E}^{0}(X, T)$ is $T$-invariant but not necessarily compact.

At the first glance, our zero-entropy set seems smaller than some "nontypical" point sets studied in Barreira and Schmeling ${ }^{(2)}$ since they proved those "non-typical" point sets have full topological entropy and full dimension property; while one can always find many typical points which are zeroentropy points, for examples, periodic points of $T$. One may guess that from the view point of the ergodic theory, $\left(X \backslash \mathcal{E}^{0}, T_{\mid X \backslash \mathcal{E}^{0}}\right)$ might be a really small subsystem. However, we have Theorem 1.

Theorem 1. Suppose $(X, T)$ is a locally expanding compact dynamical system. Then the Hausdorff dimension of the zero-entropy set vanishes, that is, $\operatorname{HD}\left(\mathcal{E}^{0}(X, T)\right)=0$.

Note that every locally expanding compact system must have positive topological entropy. Here $(X, T)$ is locally expanding if there exists $\varepsilon>0$ and $\lambda>1$ so that

$$
d(T x, T y) \geqslant \lambda d(x, y) \quad(\forall x, y \in X \text { with } d(x, y)<\varepsilon) .
$$

Theorem 1 shows that the zero-entropy set is really small from the viewpoint of topology, although it might be large from the viewpoint of ergodic theory. On the other hand, it might be still useful to describe the topological property of the substitution sequences in substitution dynamics, automatons, coding and symbolic dynamics.

The paper is organized as follows. In Section 2.1, we first review Bowen's definition of entropy for noncompact sets. Then in Section 2.2, we estimate the entropy of the zero-entropy set of a given compact dynamical system. Finally, in Section 2.3, we prove our main result (Theorem 1) in this paper. In Section 2.4, we post a question. In Section 2.5, we give an example to show why locally expanding is necessary in our result. In Section 3, we give an application of our main theorem to substitution sequences.

\section{ENTROPY AND HAUSDORFF DIMENSION}

We need Bowen's definition of entropy for non-compact sets in our proof of Theorem 1. Let us recall it. Suppose $(X, T)$ is a compact dynamical system on a compact metric space $(X, d)$.

\subsection{Bowen's Entropy for Noncompact Sets}

For any $Y \subseteq X$ not necessarily compact, Bowen defined in ref. 3 the topological entropy $h_{\mathrm{H}}(T ; Y)$ much like Hausdorff dimension, that is, with 
the "size" of a set reflecting how $T$ acts on it rather than its diameter. Here the subscript ${ }_{H}$ means the topological entropy is defined in the viewpoint of Hausdorff dimension. Let $\mathfrak{U}$ be a finite open cover of $X$. We write $E \prec \mathfrak{U}$ if $E$ is contained in some member of $\mathfrak{U}$ and $\left\{E_{i}\right\} \prec \mathfrak{U}$ if every $E_{i} \prec \mathfrak{U}$. Let $l_{T, \mathfrak{U}}(E)$ be the biggest nonnegative integer such that

$$
T^{k}(E) \prec \mathfrak{U} \quad \forall k \in\left[0, l_{T, \mathfrak{U}}(E)\right),
$$

where $l_{T, \mathfrak{U}}(E)=0$ if $E \nprec \mathfrak{U}$ and $l_{T, \mathfrak{U}}(E)=+\infty$ if $T^{k}(E) \prec \mathfrak{U}$ for all $k \geqslant 1$. Now set

$$
\operatorname{diam}_{\mathfrak{U}}(E)=\exp \left(-l_{T, \mathfrak{U}}(E)\right)
$$

and then

$$
\mathrm{D}_{\mathfrak{U}}(\mathfrak{E}, \lambda)=\sum_{i=1}^{\infty}\left(\operatorname{diam}_{\mathfrak{U}}\left(E_{i}\right)\right)^{\lambda}
$$

for any family of subsets $\mathfrak{E}=\left\{E_{i}\right\}_{1}^{\infty}$ and for any $\lambda \in \mathbb{R}_{+}$. Define the measure $M_{\mathfrak{U}, \lambda}(Y)$ by

$$
M_{\mathfrak{U}, \lambda}(Y)=\lim _{\varepsilon \rightarrow 0} \inf \left\{\mathrm{D}_{\mathfrak{U}}(\mathfrak{E}, \lambda) \mid \bigcup E_{i} \supseteq Y, \operatorname{diam}_{\mathfrak{U}}\left(E_{i}\right)<\varepsilon\right\}
$$

and then define

$$
\operatorname{dim}_{\mathrm{b}, \mathfrak{U}}(T, Y)=\inf \left\{\lambda \in \mathbb{R}_{+}: M_{\mathfrak{U}, \lambda}(Y)=0\right\}
$$

Finally, Bowen's dimension entropy of $T$ relative to $Y \subseteq X$ is given by

$$
h_{\mathrm{H}}(T ; Y)=\sup _{\mathfrak{U}}\left\{\operatorname{dim}_{\mathrm{b}, \mathfrak{A}}(T, Y)\right\},
$$

where $\mathfrak{U}$ ranges over all finite open covers of $X$. For the special case where $Y=X$, we write

$$
h_{\mathrm{H}}(T)=h_{\mathrm{H}}(T ; X) \text {. }
$$

However, since $X$ is compact, $h_{\mathrm{H}}(T)$ identically equals the usual topological entropy $h(T)$ defined by Adler et al. in ref. 1 . 


\subsection{The Entropy of the Zero-Entropy Set}

Since the zero-entropy set $\mathcal{E}^{0}(X, T)$ is not necessarily compact, we need to use Bowen's dimension entropy.

Lemma 1. Let $(X, T)$ be a compact dynamical system and $Y \subseteq X$. Then

$$
h_{\mathrm{H}}\left(T ; \bigcup_{y \in Y} \overline{\operatorname{Orb}_{+}(y)}\right)=\sup _{y \in Y}\left\{h\left(T ; \overline{\operatorname{Orb}_{+}(y)}\right)\right\} .
$$

Proof. In order to prove the lemma, we only need to show

$$
h_{\mathrm{H}}\left(T ; \bigcup_{y \in Y} \overline{\operatorname{Orb}_{+}(y)}\right) \leqslant \sup _{y \in Y}\left\{h\left(T ; \overline{\operatorname{Orb}_{+}(y)}\right)\right\} .
$$

Write

$$
\Upsilon=\bigcup_{y \in Y} \overline{\operatorname{Orb}_{+}(y)}
$$

For any $x \in \Upsilon$, there is some $\hat{y} \in Y$ such that $x \in \overline{\operatorname{Orb}_{+}(\hat{y})}$. Let $V_{T}(x)$ be the set of the weak* accumulation points of the sequence of measures $\left\{\mu_{n, x}\right\}_{n=1}^{\infty}$, where

$$
\mu_{n, x}:=\frac{1}{n} \sum_{i=0}^{n-1} \delta_{T^{i} x} \quad(n=1,2, \ldots) .
$$

Here $\delta_{T^{i} x}$ denotes the Dirac probability measure concentrated on the point $T^{i} x$. The closure of $\operatorname{Orb}_{+}(x)$ is a closed $T$-invariant set contained in $\overline{\operatorname{Orb}_{+}(\hat{y})}$. Therefore, for every measure $\mu \in V_{T}(x)$ the $\operatorname{support} \operatorname{supp}(\mu)$ is contained in $\overline{\operatorname{Orb}_{+}(\hat{y})}$. The same is true for almost every ergodic component of $\mu$, so

$$
h_{\mu}(T) \leqslant h\left(T ; \overline{\operatorname{Orb}_{+}(\hat{y})}\right) \leqslant \sup _{x \in Y}\left\{h\left(T ; \overline{\operatorname{Orb}_{+}(x)}\right)\right\} .
$$

Thus by [ref. 3, Theorem 2], $h_{\mathrm{H}}(T ; \Upsilon) \leqslant \sup _{x \in Y}\left\{h\left(T ; \overline{\operatorname{Orb}_{+}(x)}\right)\right\}$, as desired. 
Remark 1. Lemma 1 above is nontrivial because Bowen's dimension entropy $h_{\mathrm{H}}(\cdot)$ has only the countable stability (refer to ref. 3) and Adler et al. entropy $h(\cdot)$ has only the finite stability (refer to ref. 7, Theorem 7.5). But in the lemma, $\bigcup_{y \in Y} \overline{\operatorname{Orb}_{+}(y)}$ could be uncountable union of sets.

For any real number $\alpha \geqslant 0$ and for any given compact dynamical system $(X, T)$, let

$$
\mathcal{E}^{\alpha}(X, T)=\bigcup\left\{\overline{\operatorname{Orb}_{+}(x)} \mid x \in X, h\left(T ; \overline{\operatorname{Orb}_{+}(x)}\right)=\alpha\right\} .
$$

Lemma 2. Let $(X, T)$ be a compact dynamical system. Then

$$
h_{\mathrm{H}}\left(T ; \mathcal{E}^{\alpha}(X, T)\right)=\alpha \quad\left(\forall \alpha \in \mathbb{R}_{+}\right)
$$

if $\mathcal{E}^{\alpha}(X, T) \neq \varnothing$. In particular, $h_{\mathrm{H}}\left(T ; \mathcal{E}^{0}(X, T)\right)=0$.

Proof. The statement comes immediately from Lemma 1.

\subsection{Proof of Theorem 1}

Recall that a compact dynamical system $(X, T)$ is $\varepsilon$-locally expanding with skewness $\lambda$ if there exists $\varepsilon>0$ and $\lambda>1$ so that

$$
d(T x, T y) \geqslant \lambda d(x, y) \quad(\forall x, y \in X \text { with } d(x, y)<\varepsilon) .
$$

For a locally expanding dynamical system, the following relation holds:

Lemma 3 ([ref. 5, Theorem 4.5]). Let $(X, d)$ be a compact metric space and let $f: X \rightarrow X$ be an $\varepsilon$-locally expanding map with skewness $\lambda$. Then

$$
\mathrm{HD}(Y) \leqslant \frac{h_{\mathrm{H}}(f ; Y)}{\log \lambda} \quad(\forall Y \subseteq X) .
$$

Proposition 1. Let $(X, T)$ be $\varepsilon$-locally expanding with skewness $\lambda$. Then the following inequality holds:

$$
\operatorname{HD}\left(\mathcal{E}^{\alpha}(X, T)\right) \leqslant \alpha / \log \lambda \quad\left(\forall \alpha \in \mathbb{R}_{+}\right) .
$$

Proof. The statement follows easily from Lemmas 2 and 3 .

Proof of Theorem 1. As $h_{\mathrm{H}}\left(T ; \mathcal{E}^{0}(X, T)\right)=0$, by Lemma 2, the statement follows immediately from Proposition 1. 


\subsection{An Open Problem}

For a locally expanding compact dynamical system $(X, T)$, it is known that there is a unique maximal-entropy probability measure $\mu_{\max }$ such that $h_{\mu_{\max }}(T)=h(T)$. Write simply $\alpha_{\max }=h_{\mu_{\max }}(T)$. It is easily seen that

$$
\begin{aligned}
\mathcal{E}^{\alpha_{\max }}(X, T) & =\operatorname{supp}\left(\mu_{\max }\right), \\
\operatorname{HD}\left(\mu_{\max }\right) & \leqslant \operatorname{HD}\left(\mathcal{E}^{\alpha_{\max }}(X, T)\right) .
\end{aligned}
$$

Here

$$
\operatorname{HD}(\mu):=\inf \{\operatorname{HD}(B) \mid B \in \mathfrak{B}(X), \quad \mu(B)=1\}
$$

for any Borel probability measure $\mu$ supported on $X$. It is should be noted that in general

$$
\mathrm{HD}(\mu) \leqslant \mathrm{HD}(\operatorname{supp}(\mu)) .
$$

We would like to ask the following question.

Question 1. For a locally expanding compact dynamical system $(X, T)$, whether

$$
\operatorname{HD}\left(\mathcal{E}^{\alpha_{\max }}(X, T)\right)=\operatorname{HD}(X) ?
$$

and whether

$$
\operatorname{HD}\left(\mu_{\max }\right)=\operatorname{HD}\left(\mathcal{E}^{\alpha_{\max }}(X, T)\right) ?
$$

This problem is interesting in statistical physics. We conjecture that its answer is positive. In ref. 4, by choosing an equivalent "good" metric, the author proved that $\operatorname{HD}\left(\mu_{\max }\right)=\operatorname{HD}\left(\mathcal{E}^{\alpha_{\max }}(X, T)\right)=\operatorname{HD}(X)$ in the sense of the new metric. In particular, the standard metric of a symbolic system defined as in Eq. (3.3) below is "good" in our sense.

\subsection{A Further Remarks on the Condition "Locally Expanding"}

Let $S^{1}$ be the unit circle with the usual metric. Consider a rational rotation

$$
R_{\alpha}: S^{1} \rightarrow S^{1}
$$

with nonnegative rotation number $\alpha \in \mathbb{Q}$. Clearly, 


$$
\mathcal{E}^{0}\left(S^{1}, R_{\alpha}\right)=S^{1} \quad \text { and } \quad \operatorname{HD}\left(S^{1}\right)=1 .
$$

For any ergodic measure $\mu$ of $\left(S^{1}, R_{\alpha}\right)$, we have $\operatorname{HD}(\mu)=0<\operatorname{HD}\left(S^{1}\right)$. Therefore, the condition " $\varepsilon$-locally expanding with skewness $\lambda$ " is essentially needed in our Theorem 1 and Question 1.

\section{AN APPLICATION TO SUBSTITUTION SEQUENCES}

Let $A=\{0,1, \ldots, s-1\}$ be the alphabet of cardinality $s \geqslant 2 . A^{*}$ denotes the set of all finite words over $A$. A substitution $\zeta$ on $A$ is a map from $A$ to $A^{*}$ which associates each letter $i \in A, 0 \leqslant i \leqslant s-1$, to a word $\zeta(i)$ with length $|\zeta(i)|=\ell_{i}$ (assumed to be $\geqslant 2$ for one $i$ at least). Any substitution $\zeta$ induces a map from $A^{*}$ to $A^{*}$ by putting

$$
\zeta(w)=\zeta\left(w_{0}\right) \zeta\left(w_{1}\right) \cdots \zeta\left(w_{n}\right) \quad \text { if } w=w_{0} w_{1} \ldots w_{n} \in A^{*}
$$

and likewise we can define a map from $A^{\mathbb{N}}$ to $A^{\mathbb{N}}$, also denoted by $\zeta$, by

$$
\zeta(\mathrm{x})=\zeta\left(x_{0}\right) \zeta\left(x_{1}\right) \cdots \quad \text { if } \mathrm{x}=\left(x_{n}\right)_{n} \geqslant 0 \in A^{\mathbb{N}} .
$$

A substitution $\zeta$ is said to be primitive if there exists $k$ such that $\zeta^{k}(\alpha)$ contains $\beta$ for every pair $\alpha, \beta \in A$ [ref. 6, Definition V.2].

Let $\lambda>1$ be arbitrarily given. Take on $A^{\mathbb{N}}$ a topology induced by the following standard metric

$$
d(\mathrm{x}, \mathrm{y})=\lambda^{-\mathrm{n}(\mathrm{x}, \mathrm{y})} \quad \text { if } \mathrm{x}=\left(x_{i}\right)_{i} \geqslant 0 \text { and } \mathrm{y}=\left(y_{i}\right)_{i \geqslant 0} \in A^{\mathbb{N}},
$$

where

$$
\mathrm{n}(\mathrm{x}, \mathrm{y})= \begin{cases}\infty, & \text { if } \mathrm{x}=\mathrm{y} \\ \min \left\{i \mid x_{i} \neq y_{i}\right\}, & \text { if } \mathrm{x} \neq \mathrm{y}\end{cases}
$$

Notice that $\zeta: A^{\mathbb{N}} \rightarrow A^{\mathbb{N}}$ is continuous but may not onto. In the theory of substitution systems, one is interested in fixed points of $\zeta$ or $\zeta^{k}$ for $k>1$, those points are called substitution sequences defined by $\zeta$ (see, e.g., ref. 6).

Let

$$
\sigma: A^{\mathbb{N}} \longrightarrow A^{\mathbb{N}}
$$

be the standard shift defined by

$$
\left(x_{0} x_{1} x_{2} \cdots\right) \longmapsto\left(x_{1} x_{2} \cdots\right) \quad\left(\forall \mathrm{x}=\left(x_{i}\right)_{i \geqslant 0} \in A^{\mathbb{N}}\right) .
$$


Then $\left(A^{\mathbb{N}}, \sigma\right)$ is a compact positive entropy dynamical system and is locally expanding.

For any $\mathrm{x} \in A^{\mathbb{N}}$, let $\overline{\mathrm{Orb}_{+}(\mathrm{x} ; \sigma)}$ be the closure of the forward orbit $\left\{\sigma^{n}(\mathrm{x}) \mid n \in \mathbb{Z}_{+}\right\}$of $\mathrm{x}$ under $\sigma$. Then $\left(\overline{\mathrm{Orb}_{+}(\mathrm{x} ; \sigma)}, \sigma_{\mid \overline{\mathrm{Orb}_{+}(\mathrm{x} ; \sigma)}}\right)$ is a compact subsystem of $\left(A^{\mathbb{N}}, \sigma\right)$. Write

$$
S_{\zeta}=\bigcup\left\{\overline{\operatorname{Orb}_{+}(\mathrm{u} ; \sigma)} \mid \mathrm{u} \text { is a substitution sequence of } \zeta\right\} .
$$

In the study of substitution systems, one would like to know how big is $S_{\zeta}$ ? From our Theorem 1, we have the following result.

Proposition 2. Let $\zeta$ be a primitive substitution on $A$. Then $\mathrm{HD}\left(S_{\zeta}\right)=0$.

Proof. For any $\mathrm{x} \in S_{\zeta}$, it follows from [ref. 6, Proposition V.19] that

$$
h\left(\sigma ; \overline{\mathrm{Orb}_{+}(\mathrm{x} ; \sigma)}\right)=0 .
$$

Furthermore, by Theorem 1, we obtain $\operatorname{HD}\left(S_{\zeta}\right)=0$.

\section{ACKNOWLEDGMENT}

The first author is partially supported by NSF grants \#C19901029 and the second author is partially supported by NSF grants and PSCCUNY awards and the Hundred Talents Program from Academia Sinica.

\section{REFERENCES}

1. R. Adler, A. Konheim and M. McAndrew, Topological entropy, Trans. Amer. Math. Soc. 114:309-319 (1965).

2. L. Barreira, and J. Schmeling, Sets of "non-typical" points have full topological entropy and full Hausdorff dimension. Isreal J. Math. 116:29-70 (2000).

3. R. Bowen, Topological entropy for noncompact sets. Trans. Amer. Math. Soc. 184:125-136 (1973).

4. X. Dai, Existence of full-Hausdorff-dimension invariant measures of dynamical systems with dimension metrics. Arch. Math. To appear.

5. X. Dai, and Y.-P. Jiang, Distance entropy of dynamical systems on noncompact-phase spaces. Submitted.

6. M. Queffélec, Substitution Dynamical Systems-Spectral Analysis. Lect. Notes in Math. 1294 (Springer-Verlag, Berlin, New York, 1987).

7. P. Walters, An Introduction to Ergodic Theory. G.T.M. Vol. 79 (Springer-Verlag, New York, 1982). 